\title{
DIAGNÓSTICO POR IMAGEM DE HÉRNIA DISCAL HANSEN TIPO I, II E III EM CÃES
}

Lidiane da Silva Alves ${ }^{1}$

\begin{abstract}
RESUMO
Para o presente trabalho, foi realizado um levantamento bibliográfico abordando os métodos diagnósticos da hérnia discal, a qual é a discopatia mais comum em cães e está relacionada aos distúrbios neurológicos que envolvem a medula espinhal. A hérnia discal é a principal causa para a investigação imagenológica da coluna vertebral em pequenos animais. $\mathrm{O}$ diagnóstico por imagem é fundamental para obter o conhecimento da doença, da localidade da lesão e da gravidade. Os exames mais comuns realizados na rotina veterinária são a radiografia e a mielografia, por serem mais acessíveis, porém o diagnóstico preciso se dá por meio da tomografia computadorizada ou da ressonância magnética, sendo esta última a técnica primordial para as discopatias. Esses fatores, bem como sua etiologia, fisiopatologia e predisposição serão abordados também neste estudo.
\end{abstract}

Palavras-chave: disco intervertebral, radiografia, tomografia computadorizada, ressonância magnética

\section{IMAGING DIAGNOSTIC OF HANSEN TYPE I, II AND III DISC HERNIATION IN DOGS}

\begin{abstract}
For the present study was reviewed bibliography about the diagnostic methods of disc herniation, which it is the discopathy most common in dogs and it is related to neurological disorders involving the spinal cord. Disc herniation is the main cause for the imaging investigation of the spine in small animal. Diagnostic imaging is fundamental to have knowledge of the disease, the location and severity of the lesion. The most common examinations performed in veterinary medicine are radiography and myelography, because they are more accessible, but an accurate diagnosis is made through computed tomography or magnetic resonance, being the latter the primary technique to the discopathy. These factors, as well as the etiology and pathophysiology will be approached also in this study.
\end{abstract}

Keywords: intervertebral disc, radiography, computed tomography, magnetic resonance

\section{DIAGNÓSTICO POR IMAGEN DE LA HERNIA DISCAL HANSEN TIPO I, II Y III EN LOS PERROS}

\section{RESUMEN}

Para este estudio, se realizó una literatura sobre los métodos de diagnóstico de la hernia de disco, que es la enfermedad del disco más común en los perros y se relaciona con trastornos neurológicos que implican la médula espinal. La hernia de disco es la causa principal para la investigación por imagen de la columna vertebral en los animales pequeños. El diagnóstico

\footnotetext{
${ }^{1}$ Faculdade de Medicina Veterinária e Zootecnia, UNESP - Campus Botucatu.
} 
por imagen es esencial para la comprensión de la enfermedad, la localización de la lesión y la gravedad. Las pruebas más comunes que se realizan en la medicina veterinaria son la radiografía y la mielografía, debido a que son más asequibles, pero el diagnóstico exacto es a través de la tomografía computarizada o resonancia magnética, siendo esta última la principal técnica de discopatías. Estos factores, así como su etiología, fisiopatología y la predisposición también serán abordados en este trabajo.

Palabras claves: disco intervertebral, radiografía, tomografía computarizada, resonancia magnética

\section{INTRODUÇÃO}

A degeneração do disco intervertebral e, consequentemente, a hérnia discal são as causas mais comuns de alterações neurológicas em cães (1-6). Tais causas podem levar a compressão da medula espinhal ou das raízes nervosas $(2,6)$. Essa alteração ocorre em raças condrodistróficas e não condrodistróficas (7).

A apresentação clínica é variável, dependendo da localização da lesão, do volume de material no interior do canal $(1,2,5,8)$, da velocidade com que este material é ejetado $(2,5)$ e da duração da lesão $(1,5,8)$. Os sinais clínicos podem ser ataxia e paresia, nos casos brandos, e paralisia com ou sem a perda da função sensitiva caudalmente à lesão, nos casos mais graves $(1,9)$.

O diagnóstico baseia-se na resenha, anamnese e exame neurológico $(1,8)$, além da realização de exames complementares para o diagnóstico preciso e definitivo do local de extrusão ou protrusão do disco herniado (8), como a radiografia simples e contrastada (mielografia), a tomografia computadorizada e mielotomografia, e a ressonância magnética (7). Embora a radiografia seja o exame de primeira escolha, é um método não confirmatório para o diagnóstico de hérnia de disco intervertebral (10). A mielografia e a ressonância magnética são exames de diagnóstico definitivo de cães com suspeita de herniação $(3,9,10)$. Uma imagem precisa pode estabelecer a presença e a gravidade da doença do disco intervertebral, permitindo que os clínicos determinem o prognóstico e realizem o tratamento (6).

O tratamento depende do tipo e grau da lesão (8), havendo muitas controversas quanto à determinação de uma técnica específica para cada caso (2). O tratamento pode ser clínico conservativo, utilizando analgésicos e anti-inflamatórios associados ao repouso absoluto sob confinamento (1,2), ou então cirúrgico, utilizando técnicas descompressivas como a hemilaminectomia e laminectomia $(2,7,9)$, a facetectomia (7), a fenestração ventral, a fenestração lateral (1) e ainda a pediculectomia (11). Outras formas de tratamento que vem sendo bastante utilizadas na medicina veterinária são acupuntura $(12)$, fisioterapia $(13,14)$, eletroacupuntura $(15,16)$ e a ozonioterapia (17). Novas técnicas têm sido desenvolvidas para o tratamento da degeneração do disco intervertebral em cães, como o transplante de condrócitos discais (18).

A suspeita de doença do disco intervertebral é uma das mais importantes indicações para a obtenção de imagens da coluna vertebral em pequenos animais (6). Diante disso, o objetivo deste estudo foi avaliar os métodos de diagnóstico por imagem em cães com hérnia discal para diagnosticar corretamente a doença e então proceder ao respectivo tratamento.

\section{Etiologia e fisiopatologia}


Em geral, as hérnias discais estão associadas a degeneração dos discos intervertebrais (DDIV), causando a protrusão ou extrusão do material discal para o interior do canal vertebral, sendo de forma espontânea ou secundária a um trauma, levando a compressão da medula espinhal ou das raízes nervosas (19).

A estrutura anatômica dos discos intervertebrais (DIVs) podem explicar as ocorrências mais comuns de protrusão ou extrusão pelo fato do anel fibroso ser mais espesso nas porções ventral e laterais, da sua localização dorsalmente excêntrica do núcleo pulposo e da maior fragilidade do ligamento longitudinal dorsal em relação ao ligamento longitudinal ventral, assim promovendo a herniação dorsal. Além da compressão dorsal da medula espinhal, podem ocorrer compressões ventral e lateral com aprisionamento do nervo espinhal (20). Outros fatores podem influenciar a hérnia de DIV, como a genética, o comprimento da coluna vertebral, o grau de aptidão muscular e o peso corporal (21).

\section{Hansen tipo I ou extrusão}

Na extrusão do DIV ocorre a ruptura do anel fibroso dorsal (22) devido a degeneração ou metaplasia condroide e, então, o material do núcleo pulposo sai e vai para o interior do canal vertebral (23). A degeneração condroide é caracterizada pela desidratação e calcificação do núcleo pulposo (6), podendo ser visualizados precocemente nas radiografias (23).

Esse tipo de hérnia, envolve a concentração de glicosaminoglicano, perda de conteúdo líquido e de conteúdo proteoglicano, e aumenta o conteúdo de colágeno. Com isso, o disco torna-se mais cartilaginoso e o núcleo torna-se mais granular, normalmente mineralizados, e perde sua qualidade de absorção de choque hidroelástico (19).

Ocorrem normalmente de forma aguda (6) lesionando as estruturas anatômicas da região e comprimindo a medula, podendo ocorrer hemorragia extradural ou intradural e tumefação da medula espinhal causando início agudo de para- ou tetraparesia (24). A extrusão discal também pode ocorrer de forma lenta e progressiva, produzindo paresia que se torna mais severa após vários dias ou tem curso crônico e irregular. A lesão traumática grave pode levar a alterações vasculares secundárias como a mielomalácia, que pode ser ascendente ou descendente (23).

Nesse tipo de hérnia, quando ocorre de forma lenta, o material discal pode extravasar para ambos os lados do ligamento longitudinal dorsal e o animal pode apresentar déficit maior num membro em relação ao outro (23).

É comum nas raças condrodistróficas (Dachshund, Pequinês, Beagle, Poodle, Shih Tzu, Lhasa Apso, Basset Hound e Welsh Corgis) $(5,6,19)$ e normalmente em animais jovens (24), com idade variando de dois a sete anos, com o pico de incidência aos quatro e cinco anos (19). Cerca de $62 \%$ a $92 \%$ dos cães não condrodistróficos pesando mais que $20 \mathrm{Kg}$ possuem extrusão do DIV toracolombar (22).

\section{Hansen tipo II ou protrusão}

A protrusão do DIV está tipicamente associada à degeneração fibrosa ou metaplasia fibroide $(6,19,22,23)$. À medida que o animal vai envelhecendo, o núcleo é acometido pela desidratação, no lugar de ser substituído por cartilagem hialina, é substituído por fibrocartilagem (20), sem que o DIV mineralize, como acontece na degeneração condroide $(22,23)$, não podendo ser vistos nas radiografias de rotina da coluna vertebral (23).

$\mathrm{O}$ anel fibroso pode sofrer ruptura parcial das fibras $(22,23)$, se estirar ou hipertrofiar (6), não havendo a saída do material nuclear e sim uma saliência do DIV que comprime a medula espinhal (23). O processo de protrusão ocorre de forma crônica (6), promovendo paresia lentamente progressiva que evolui em semanas ou meses (23). 
A maior ocorrência de protrusão é em pacientes idosos (6), com idade variando de oito a dez anos (19) e em raças de grande porte não condrodistróficas $(6,19)$, mostrando maior incidência em cães da raça Pastor Alemão. Predileção sexual não tem sido reportada, embora alguns trabalhos relatem que machos e fêmeas castradas apresentaram alto risco de desenvolvimento de hérnia de DIV que fêmeas não castradas. A degeneração fibroide acomete apenas um pequeno número de DIV (22), sendo os locais mais comuns de T12-T13 (23).

\section{Hansen tipo III}

A hérnia Hansen tipo III se caracteriza pela herniação discal traumática não degenerativa $(6,19)$. Se um disco com o núcleo pulposo adequadamente hidratado é colocado sobre extremo estresse, o anel fibroso dorsal pode-se romper, e então o material discal saudável é ejetado para o canal vertebral causando contusão medular. Como esse material está hidratado, ele difunde-se na gordura peridural deixando apenas às alterações secundárias atribuíveis a contusão medular aguda com pouca ou nenhuma compressão da medula espinhal (19).

\section{DIAGNÓSTICO POR IMAGEM}

\section{Radiografia simples}

Os sinais radiográficos de hérnia discal são observados pelo estreitamento do espaço intervertebral $(6,22,23,25,26)$, do forame intervertebral e do espaço articular intervertebral dorsal $(6,24)$, opacificação do forame intervertebral $(6,22,25)$ e cuneiformização dos corpos vertebrais contíguos $(22,25,26)$.

Em extrusão discal, que ocorre a calcificação dos DIVs previamente, são bem visíveis em radiografias simples $(6,23)$ pelo material discal calcificado dentro do anel dorsal ou no canal medular (23). Já em protrusão discal, onde não há mineralização do material de DIV, pode ser identificado como uma estrutura de densidade semelhante à dos tecidos moles, verificando-se que este achado é mais facilmente detectado ao nível da coluna vertebral lombar, já que não há sobreposição das costelas aos forames intervertebrais (6).

Associado às hérnias Hansen tipos I e II podem ser identificados sinais radiográficos de osteófitos vertebrais (20), espondilose deformante e esclerose vertebral $(6,20)$. Os osteófitos vertebrais são, geralmente, ventrais e laterais; se dorsais, eles podem causar estenose do canal vertebral (20).

\section{$\underline{\text { Mielografia }}$}

A mielografia é um estudo contrastado usado para avaliar a medula espinhal pela injeção de meios de contrastes não iônicos no espaço subaracnoide. É utilizada para identificar sítios de compressão da medula espinhal devido ao edema, material discal, anormalidade vertebral ou neoformações (27). Contudo, a mielografia deve ser realizada somente após um exame radiográfico simples de boa qualidade (28).

Para a realização da mielografia, o animal deve ser submetido a anestesia geral e então injetar o meio de contraste entre as vértebras $\mathrm{C} 1-\mathrm{C} 2$ com o paciente em decúbito lateral com hiperflexão da cabeça ou entre L5-L6 com o paciente em decúbito esternal ou lateral com a coluna lombar fletida e os membros posteriores estendidos cranialmente abrindo o espaço intervertebral (27). 
Os meios de contrastes injetados na medula espinhal são iohexol ou iopamidol utilizando doses específicas para cada local de punção, sendo na região de cisterna as doses de $0,3 \mathrm{ml} / \mathrm{kg}$ para avaliar o segmento cervical e $0,45 \mathrm{ml} / \mathrm{kg}$ para o segmento toracolombar; assim como na região lombar que se utiliza $0,45 \mathrm{ml} / \mathrm{kg}$ para avaliar o segmento cervical e $0,3 \mathrm{ml} / \mathrm{kg}$ o segmento toracolombar (27).

Se o paciente apresenta paralisia aguda e com uma única lesão demonstrada no exame simples, torna-se desnecessária a mielografia, a menos que os achados neurológicos não sejam compatíveis com o diagnóstico radiográfico (29), pois a mielografia é um procedimento invasivo podendo piorar o estado neurológico de alguns animais $(6,30)$. Podem ocorrer convulsões durante a recuperação, principalmente se o meio de contraste adentrar ao crânio.

A mielografia não permite um diagnóstico patológico definitivo das lesões espinhais, mas certamente permite a identificação do local das lesões ao longo da espinha e também a localização da lesão em relação à medula e meninges (31). Durante a interpretação da mielografia, deve se estar ciente que podem existir artefatos mielográficos, como injeção epidural ou subdural acidental que podem dificultar o diagnóstico. Se houver ausência de alterações na mielografia, o paciente deve ser submetido a outros exames de imagem, como a TC ou a RM.

Os sinais mielográficos da hérnia discal são compressão extradural da medula espinhal com deslocamento e modificação da largura da coluna de contraste, diminuição do espaço subaracnoide e sua opacificação nas projeções lateral ou ventrodorsal, estreitamento do espaço de DIV (6) e o desvio dorsal do espaço subaracnoide ventral (Figura 1) ao nível da herniação discal nas projeções laterais $(6,26)$. Quando a protrusão de DIV é ligeiramente lateral à linha média, a aparência observada da coluna do meio de contraste pode ser fendida ou bifurcada, apresentando linha dupla de contraste na projeção lateral. É visualizada também uma massa epidural com localização ventral ou ventrolateral que causa o deslocamento da medula espinhal em sentido dorsal (6).

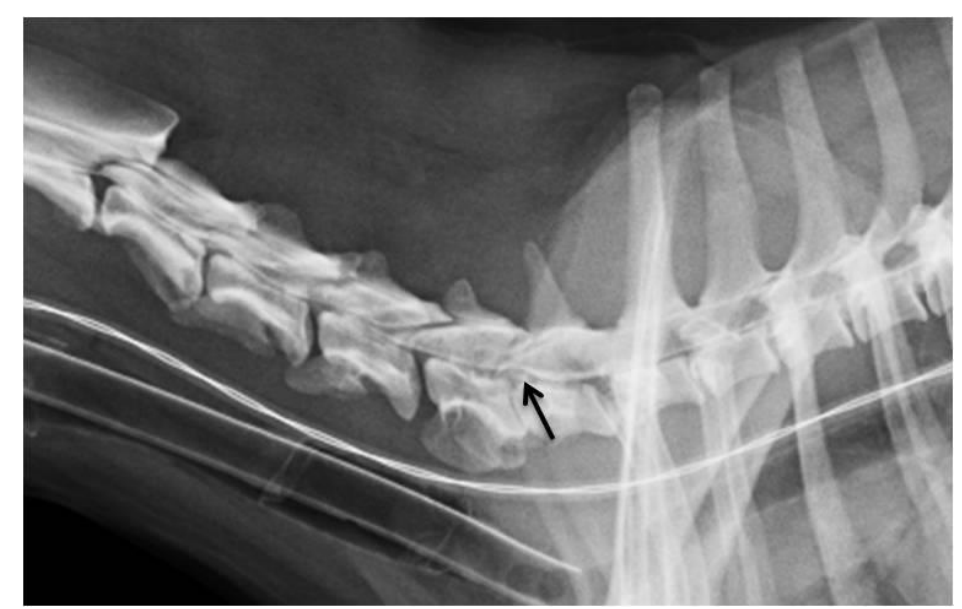

Figura 1. Incidência lateral direita de mielografia cervicotorácica de um cão sem raça definida, 7 anos, macho, com histórico de trauma por mordedura em cervical e apresentando tetraplegia. Nota-se deslocamento dorsal da coluna de contraste medular ventral (seta) ocasionada por proliferação óssea intervertebral de C6-C7 (Imagens do setor de Radiologia Veterinária da FMVZ-UNESP, Botucatu).

Existem alguns problemas técnicos da mielografia que podem comprometer a qualidade do exame e, consequentemente, o diagnóstico, como a distribuição inadequada do meio de contraste, administração errônea do meio de contraste na epidural e posicionamento radiográfico inadequado. Assim como outros fatores anatomopatológicos que podem dificultar a avaliação, como um deslocamento atípico do material discal e tumefação da medula espinhal (28). 


\section{$\underline{\text { Epidurografia }}$}

Em pacientes com o saco dural curto ou pequeno, o exame de mielografia torna-se inviável para a avaliação, sendo necessária a realização de outros procedimentos radiográficos contrastados, como a epidurografia ou discografia (32). Em sua maioria, essa técnica está direcionada para a avaliação da região lombossacra da coluna vertebral (33).

A epidurografia é realizada mediante a injeção do meio de contraste no espaço epidural ventral $(6,31)$, por uma agulha inserida no canal vertebral da junção lombossacra $(6,31,32)$ ou entre a primeira e a segunda vértebras caudais (31). A dose do contraste é similar ao da mielografia (32) ou então numa adição de 1 a $5 \mathrm{ml}$ de meio de contraste (33), assim como as projeções radiográficas (32). O desvio dorsal do aspecto ventral do espaço epidural (Figura 2) e a interrupção completa da coluna de contraste cranial constituem achados à epidurografia mais condizentes com compressão medular (6).

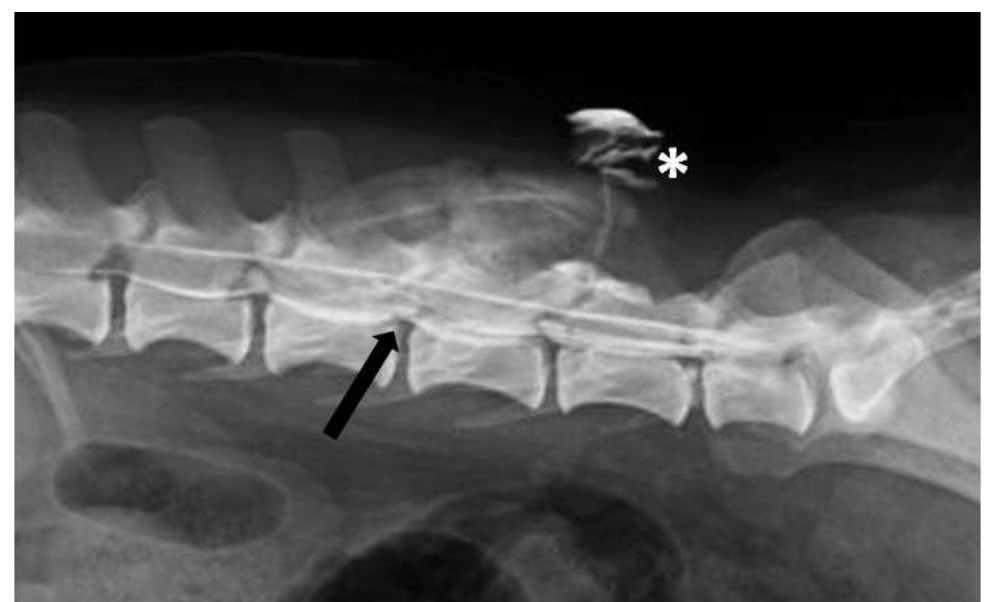

Figura 2. Epidurografia da coluna lombossacra de um cão Boxer, 10 anos, macho, apresentando paraparesia ambulatória. Neste exame, nota-se ondulações da coluna de contraste ventral (seta) representada pelo meio contraste na região epidural. Houve extravasamento do meio de contraste na musculatura dorsal (asterisco) (Imagens do setor de Radiologia Veterinária da FMVZ-UNESP, Botucatu).

\section{Discografia}

A discografia é realizada como um procedimento complementar da mielografia ou da epidurografia e provê de um excelente método de avaliação para os discos da região lombossacra (33). Para o exame, é posicionada uma agulha de 20 ou 22 gauge pelo canal vertebral, transpassando o anel fibroso até o núcleo pulposo. A dose do contraste utilizada é de $0,1 \mathrm{ml}$ em um disco normal, pois há uma resistência na aplicação a partir desse volume, e de 0,2 a $0,3 \mathrm{ml}$ em um disco degenerado. Em casos de ruptura do anel fibroso, o meio de contraste deve extravasar para o canal vertebral (32).

\section{Tomografia computadorizada}

A TC é uma ferramenta de diagnóstico sensível que pode ser utilizada como um adjunto da mielografia ou como um procedimento de diagnóstico único para, assim, evitar os efeitos secundários da mielografia (22). Entretanto, alguns autores (25) relatam que, para se obter bons resultados, é necessário ter localizado previamente a área da lesão mediante um exame neurológico e as correspondentes radiografias da coluna vertebral.

A vantagem da TC sobre a radiografia é que esta técnica tem a capacidade de produzir imagens multiplanares (dorsal, sagital e transversal) e em três dimensões (3D) (34). Além do 
mais, a resolução de contraste superior da TC permite a detecção de hérnia com material discal não mineralizado (Figura 3) e de baixa densidade mineral (6).

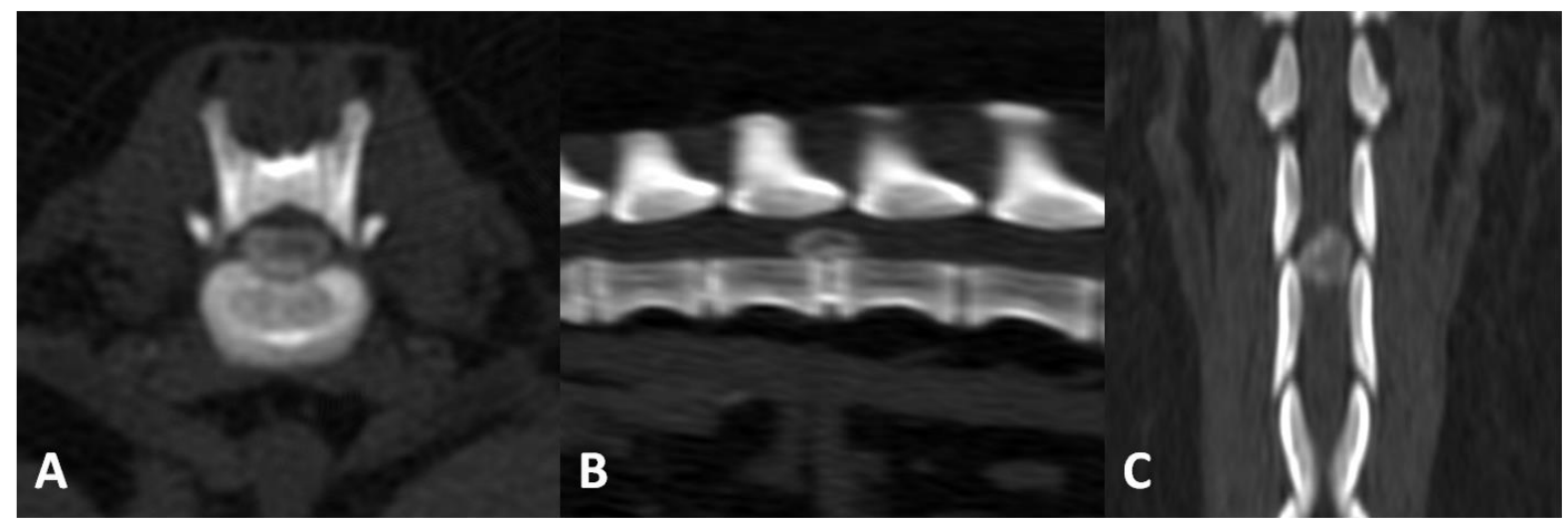

Figura 3. Tomografia computadorizada sem administração de contraste em janela óssea nos cortes (A) transversal, (B) sagital e (C) dorsal, de um cão Dachshund, 4 anos, fêmea, com paraparesia não ambulatória. Observa-se material hiperatenuante, bem definido no canal medular entre as vértebras T13-L1 compatível com extrusão do disco intervertebral mineralizado (Imagens do setor de Radiologia Veterinária da FMVZ-UNESP, Botucatu)

No exame tomográfico simples, podem ser observados o fenômeno de vácuo e as coleções de gás no interior do espaço intervertebral, devido à liberação de gases, principalmente nitrogênio, a partir de tecidos adjacentes e acúmulo dentro de fissuras do disco. O sinal de vácuo indica degeneração discal, mas não compressão da medula espinhal (24).

Para o exame de mielotomografia, injeta-se meio de contraste iodado no espaço subaracnoide em uma dose menor que para a mielografia radiográfica (19). Nesta técnica, quando há herniação discal, observa-se a compressão e o deslocamento da medula espinhal, a compressão do espaço epidural e material de densidade de tecidos moles no local da compressão (Figura 4).
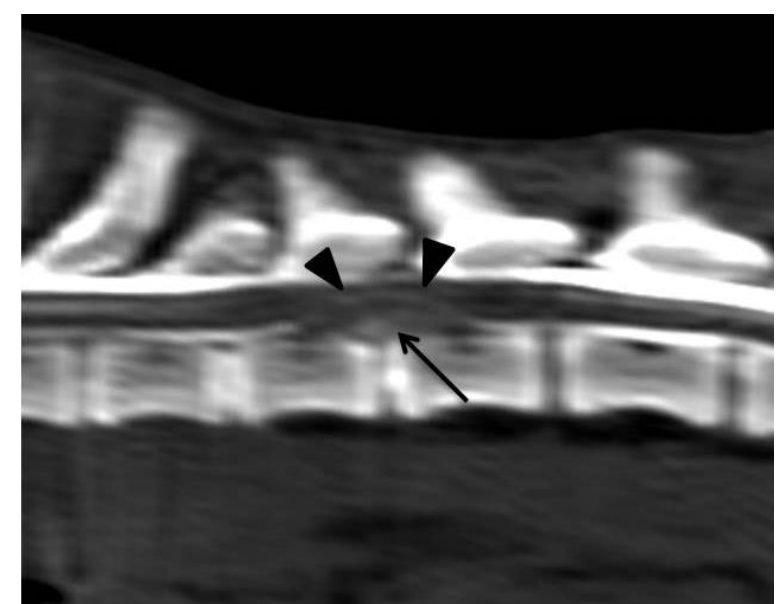

Figura 4. Corte sagital em janela óssea de um exame mielotomográfico realizado em um cão sem raça definida, 4 anos, macho, apresentando paraplegia de início agudo. É possível notar acentuado deslocamento da coluna de contraste medular ventral (pontas de setas) provocada por material de tecidos moles, compatível com extrusão discal disseminada entre T11-T12 (Imagens do setor de Radiologia Veterinária da FMVZ-UNESP, Botucatu).

$\mathrm{Na}$ avaliação tomográfica da DDIV pode-se observar hemorragia como um material hiperatenuante se estendendo cranial e caudalmente do local de compressão, edema como 
uma área hipoatenuante, preenchimento intramedular por contraste sugerindo mielomalácia e tumefação da medula espinhal (24).

\section{Ressonância magnética}

A RM é considerada o melhor método de diagnóstico para a avaliação da degeneração de DIV (22) e para gerar imagens da medula espinhal, do espaço epidural, dos DIVs e dos ligamentos espinhais (25), ou qualquer tecido que possua grandes quantidades de moléculas de água. Este método imagenológico tem como vantagem adquirir imagens primárias em qualquer plano e não necessitar de injeções de meio de contraste no espaço subaracnoide, pois o próprio LCR funciona como tal (35). Como desvantagem, o exame de RM possui um custo relativamente caro e sua técnica é limitada na avaliação mais detalhada dos ossos (36).

A RM é capaz de mostrar todos os aspectos da lesão do DIV, como o estágio de desidratação do DIV, descobrir se um DIV está somente abaulado ou rompido, a localização de qualquer material discal livre, a magnitude da compressão e o deslocamento da medula espinhal, a presença de hemorragia e edema relacionados (36) e, principalmente, o tipo de degeneração discal, se extrusão ou protrusão (22).

O núcleo pulposo normal tem um sinal hiperintenso comparado ao anel fibroso. Quando esse núcleo perde a hidratação, o sinal torna-se menos intenso e deve aparecer iso- ou hipointenso em relação ao anel fibroso. Em extrusões discais o material degenerado aparece primariamente como uma lesão hipointensa em torno da medula espinhal na imagem ponderada em T2 no corte sagital (Figura 5). Na hérnia Hansen tipo III, que ocorre a ruptura traumática de um núcleo pulposo sadio, resulta em um material hiperintenso dentro ou ao redor da medula espinhal, pois o material nuclear encontra-se normalmente hidratado (37).

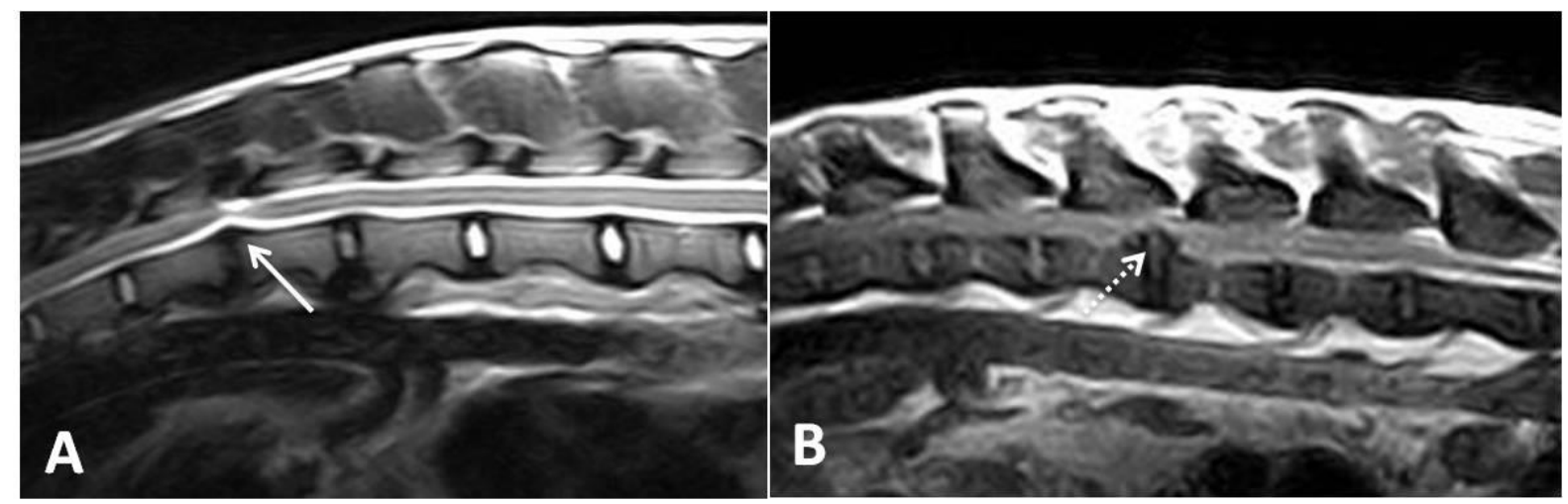

Figura 5. Ressonância magnética em plano sagital. (A) Imagem em T2 de um cão sem raça definida, 6 anos, macho, com paralisia não ambulatória, demonstrando deslocamento dorsal do trajeto medular provocada por pequeno material hipointenso entre T12-T13 compatível com protrusão discal. (B) Imagem em T1 de um cão Poodle, 6 anos, fêmea, apresentando paraparesia. Nota-se a presença de material de baixa intensidade de sinal entre L2-L3 (seta tracejada) deslocando a medula dorsalmente compatível com extrusão discal (Imagens do setor de Radiologia Veterinária da FMVZ-UNESP, Botucatu)

Normalmente, as extrusões agudas estão relacionadas à hemorragia intra- ou extramedular e edema intramedular na região da ruptura discal (36), sendo que a hemorragia resulta do dano ou ruptura do seio venoso vertebral ventral. A combinação de material discal herniado e de hemorragia resulta em uma intensidade de sinal heterogêneo nas imagens ponderadas em T2. É comum observar hipertrofia do ligamento longitudinal dorsal nas hérnias Hansen tipo II (37). Contudo, o ligamento longitudinal dorsal, que faz parte do 
assoalho do canal vertebral, pode ser mais proeminente em alguns animais podendo, então, ser confundido com protrusão discal (36).

O corte transversal fornece importante informação sobre a localização e a quantificação da compressão medular pelo material herniado. Herniação discal dorsal são as mais ocorridas, sendo as ventrais raras e contribuem ou resultam em espondilose deformante. Pode ocorrer a herniação lateral, com menor frequência, e não deve acometer a medula espinhal, porém resulta na compressão e no impacto de nervos periféricos (37).

Em herniações crônicas, o material discal dentro ou ao redor da medula espinhal deve realçar após a administração do contraste endovenoso, devido ao aumento da vascularização dentro do material e/ou associado à inflamação. Em alguns casos, o realce de contraste pode ocorrer em região periférica do material discal (37).

\section{CONSIDERAÇÕES FINAIS}

O diagnóstico por imagem é fundamental para se ter conhecimento da enfermidade, da localização da lesão e, como na tomografia computadorizada e ressonância magnética, o grau da lesão. A radiografia simples é a de primeira escolha para se tentar diagnosticar a discopatia, devido sua fácil disponibilidade, porém ela não é capaz de confirmar a doença ou a gravidade da lesão, e sim mostrar se há alguma alteração radiográfica que indica uma discopatia. Outra modalidade de fácil disponibilidade e indicadora do local da lesão é a mielografia, porém oferece riscos ao animal, assim como a discografia e epidurografia, que são menos realizadas. No Brasil, a ressonância magnética e a tomografia computadorizada ainda são de difícil acesso, estando disponíveis apenas em algumas capitais e em poucas cidades do interior. Nos países desenvolvidos, essas duas modalidadessão consideradas como primordiais, descartando-se a radiografia e mielografia na investigação de discopatias. Contudo, o diagnóstico obtido por essas técnicas permite uma correta abordagem terapêutica, sendo ela conservativa ou cirúrgica, e, consequentemente, o prognóstico. É válido lembrar que a anamnese, o exame clínico e neurológico e exames laboratoriais jamais devem ser dispensados, pois é por meios destes que o paciente é direcionado ao diagnóstico por imagem.

\section{REFERÊNCIAS}

1. Toombs JP, Bauer MS. Afecção do disco intervertebral. In: Slatter D. Manual de cirurgia de pequenos animais. 2a ed. São Paulo: Manole; 1998. v.1, p.1286-305.

2. Arias MVB, Nishioka CM, Garcia CO, Reia AZ, Baraúna Júnior D, Marcasso RA. Avaliação dos resultados clínicos após cirurgia descompressiva em cães com doença de disco intervertebral. Arq Bras Med Vet Zootec. 2007;59:1445-50.

3. Hecht S, Thomas WB, Marioni-Henry K, Echandi RL, Matthews AR, Adams WH. Myelography vs. computed tomography in the evaluation of acute thoracolumbar intervertebral disk extrusion in chondrodystrophic dogs. Vet Radiol Ultrasound. 2009;50:353-9.

4. Berknut N, Grinwis G, Pickee E, Auriemma E, Lagerstedt AS, Hagman R, et al. Reliability of macroscopic grading of intervertebral disk degeneration in dogs by use of the Thompson system and comparison with low-field magnetic resonance imaging findings. Am J Vet Res. 2011;72:899-904. 
5. Santos RP, Mazzanti A, Beckmann DV, Berté L, Ripplinger A, Polidoro Neto D, et al. Recuperação funcional em cães com doença do disco intervertebral toracolombar sem percepção à dor profunda: 37 casos (2002-2010). Pesqui Vet Bras. 2011;31:345-9.

6. Widmer WR, Thrall DE. Doença do disco intervertebral em cães e gatos, mielografia e doença medular. In: Thrall DE. Diagnóstico de radiologia veterinária. 5a ed. Rio de Janeiro: Elsevier; 2010. p.194-209.

7. Santini G, Mazzanti A, Beckmann DV, Santos RP, Pelizzari C, Polidoro D, et al. Doença do disco intervertebral cervical em cães: 28 casos (2003-2008). Pesqui Vet Bras. 2010;30:659-64.

8. Ferreira MMG, Avante MP, Rosa BRT, Martins IS, Zangirolami Filho D, Benedette MF. Discopatia toracolombar. Rev Cient Eletronica Med Vet. 2008;6:1-5.

9. Schroeder R, Pelsue DH, Park RD, Gasso D, Bruecker KA. Contrast-enhanced CT for localizing compressive thoracolumbar intervertebral disc extrusion. J Am Anim Hosp Assoc. 2011;47:203-9.

10. Robertson I, Thrall DE. Imaging dogs with suspected disc herniation: pros and cons of myelography, computed tomography, and magnetic resonance. Vet Radiol Ultrasound. 2011;52:81-4.

11. Lubbe AM, Kirberger RM, Verstraete FJM. Pediculectomy for thoracolumbar spinal decompression in the dachshund. J Am Anim Hosp Assoc. 1994;30:233-8.

12. Janssens LA. Acupuncture for the treatment of thoracolumbar and cervical disc disease in the dog. Probl Vet Med. 1992;4:107-16.

13. Ferreira AJA, Correia JHD. Thoracolumbar disc disease in 71 paraplegic dogs: influence of rate of onset and duration of clinical signs on treatment results. J Small Anim Pract. 2002;43:158-63.

14. Kathmann I, Cizinauskas S, Doherr MG, Steffen F, Jaggy A. Daily controlled physiotherapy increases survival time in dogs with suspected degenerative myelopathy. $\mathrm{J}$ Vet Intern Med. 2006;20:927-32.

15. Han H-J, Yoon H-Y, Kim J-Y, Jang H-Y, Lee B, Choi SH, et al. Clinical effect of additional electroacupuncture on thoracolumbar intervertebral disc herniation in 80 paraplegic dogs. Am J Chin Med. 2010;38:1015-25.

16. Joaquim JGF, Luna SPL, Brondani JT, Torelli SR, Rahal SC, Freitas FP. Comparison of decompressive surgery, electroacupuncture, and decompressive surgery followed by electroacupuncture for the treatment of dogs with intervertebral disk disease with longstanding severe neurologic deficits. J Am Vet Med Assoc. 2010;236:1225-9.

17. Han H-J, Kim J-Y, Jang H-Y, Lee B, Yoon J-H, Jang S-K, et al. Fluoroscopic-guided intradiscal oxygen-ozone injection therapy for thoracolumbar intervertebral disc herniations in dogs. In Vivo. 2007;21:609-14. 
18. Ganey T, Libera J, Moos V, Alasevic O, Fritsch K-G, Meisel HJ, et al. Disc chondrocyte transplantation in a canine model: a treatment for degenerated or damaged intervertebral disc. Spine. 2003;28:2609-20.

19. Mai W. Magnetic resonance imaging and computed tomography features of canine and feline spinal cord disease. In: Thrall DE. Textbook of veterinary diagnostic radiology. $6 \mathrm{a}$ ed. St. Louis: Elsevier; 2013. p.194-204.

20. Weisbrode SE. Ossos e articulações. In: McGavin MD, Achary JF. Bases da patologia em veterinária. 4a ed. Rio de Janeiro: Elsevier; 2009. p.1102-3.

21. Simpson TS. Intervertebral disc disease. Vet Clin North Am Small Anim Pract. 1992;22:889-97.

22. Costa RC. Spinal diseases. Vet Clin North Am Small Anim Pract. 2010;40:859-69.

23. Chrisman CL. Neurologia dos pequenos animais. 1a ed. São Paulo: Roca; 1985.

24. Seiler G, Kinns J, Dennison S, Saunders J, Schwarz T. Vertebral column and spinal cord. In: Schwarz T, Saunders J. Veterinary computed tomography. Iowa: Wiley-Blackwell; 2011. p.223-4.

25. Fernández T, López M. Diagnóstico por imagen de la enfermedad discal intervertebral. Rev AVEPA. 2004;24:17-25.

26. Kevin KJ, McAllister H. Radiologia e ultra-sonografia do cão e do gato. 3a ed. Barueri: Manole; 2005.

27. Ayres S. Special imaging procedures. In: Ayres S. Small animal radiographic techniques and positioning. West Sussex: Wiley Blackwell; 2012. p.233-6.

28. Lamb CR. Common difficulties with myelographic diagnosis of acute intervertebral disc prolapse in the dog. J Small Anim Pract. 1994;35:549-58.

29. Farrow CS. Veterinária: diagnóstico por imagem do cão e do gato. 1a ed. São Paulo: Roca; 2005. p.251-305.

30. Braund KG. Moléstia do disco intervertebral. In: Bojrab JM. Mecanismos da moléstia na cirurgia dos pequenos animais. 2a ed. São Paulo: Manole; 1996. p.1104-16.

31. Brawner WR, Hathcock JT. Neurorradiologia. In: Slatter D. Manual de cirurgia de pequenos animais. 2a ed. São Paulo: Manole; 1998. v.1, p.459-60.

32. Lang J. Spine - lumbosacral region and cauda equine syndrome. In: Barr FJ, Kirberger RM. BSAVA manual of canine and feline musculoskeletal imaging. Dorset: Replika Press PVT; 2006. p.260-1.

33. Barthez PY, Morgan JP, Lipsitz D. Discography and epidurography for evaluation of the lumbosacral junction in dogs with cauda equine syndrome. Vet Radiol Ultrasound. 1994;35:152-7.

Alves, LS. Diagnóstico por imagem de hérnia discal Hansen tipo I, II e III em cães. Vet. e Zootec. 2018 mar.; 25(1): 010-021. 
34. Kinns J, Malinowski R, McEvoy F, Scharz T, Zwingenberger A. Special software applications. In: Schwarz T, Saunders L. Veterinary computed tomography. West Sussex: John Wiley and Sons; 2011. p.70-1.

35. Gavin PR. Basic physics. In: Gavin PR, Bagley RS. Pratical small animal MRI. Iowa: Wiley-Blackwell; 2009. p.4-7.

36. Elliott I, Skerritt G. Using MRI in clinical veterinary practice. In: Elliott I, Skerritt G. Handbook of small animal MRI. Iowa: Wiley-Blackwell; 2010. p.98-105.

37. Bagley RS, Gavin PR, Holmes SP. Veterinary clinical MRI: diagnosis of spinal disease. In: Gavin PR, Bagley RS. Pratical small animal MRI. Iowa: Wiley-Blackwell; 2009. p.137-40.

Recebido em:

Aceito em: 